\title{
Análisis comparativo entre hemodiafiltración en línea frente a hemodiafiltración con reinfusión endógena: parámetros clínico-técnicos e inflamatorios
}

\author{
Carmen Ramírez Moreno*, Irene Torollo Luna*, Elvira Esquivias de Motta**, Ma Dolores López Zamorano*, \\ Concha Guisado Segador*, Jesús Muñoz Poyato*, Francisco Salas Cardador*, Francisco Arévalo Salamanca*, \\ Mateo Alcántara Crespo*
}

Enfermeras/os* Centro Periférico de Diálisis Perpetuo Socorro

FEA Nefrología**. Servicio de Nefrología

Unidad de Gestión Clínica de Nefrología. Hospital Universitario Reina Sofía. Córdoba

\section{Resumen}

Introducción: El tratamiento con hemodiálisis produce "per se" un proceso inflamatorio crónico en el paciente. Diferentes técnicas alternativas como la hemodiafiltración "en línea", mejoran entre otros aspectos, este perfil inflamatorio. En esta misma línea de investigación están apareciendo nuevas técnicas convectivas que sobre el papel podrían mejorar a la hemodiafiltración "en línea". La técnica HFR-SUPRA o hemodiafiltración con reinfusión endógena utiliza un dializador con doble cámara más un cartucho de resina, en el que el propio líquido ultrafiltrado del paciente es reinfundido posteriormente a su regeneración en este cartucho de resina.

El objetivo de nuestro estudio fue evaluar las diferencias entre la técnica de hemodiafiltración con reinfusión endógena y la hemodiafiltración "en línea", en cuanto a parámetros clínicos, inflamatorios y adecuación de la diálisis.

Material y métodos: Se incluyeron 16 pacientes, de edad media $61,6 \pm 14,5$ años, sometidos a las dos técnicas durante períodos de 8 semanas, según el siguiente esquema: hemodiafiltración con reinfusión endógena -1 , hemodiafiltración "en línea"-1, hemodiafiltración con reinfusión endógena -2 , hemodiafiltración "en línea" -2. Todos los pacientes estuvieron 8 semanas previas con hemodiálisis de alto flujo. Se recogieron parámetros técnicos (presión en las líneas arterial y venosa, flujo de sangre, litros tratados, volumen de in-

Correspondencia:

Carmen Ramírez Moreno.

Centro Periférico de Diálisis

Perpetuo Socorro, 12. 14012- CóRD0BA

E-mail: cramirezmdue@gmail.com fusión, necesidades de heparina), clínicos (presión arterial sistólica y diastólica pre y post-diálisis, peso seco, ganancia interdiálisis, incidencias durante la sesión) y de laboratorio (eliminación de $32 \mathrm{~m}$, hemoglobina, hematocrito, albúmina) y parámetros inflamatorios. También se analizó la duración del montaje, mediante la media de tiempo en minutos, de 10 sesiones seguidas con ambas técnicas.

Resultados: No hubo diferencias entre ambas técnicas en los parámetros clínicos recogidos: presión arterial sistólica y diastólica pre y post-diálisis, peso seco, ganancia interdiálisis, incidencias durante la sesión. Tampoco hubo diferencias significativas en las necesidades de heparina ni en el Kt/V. Los valores de hemoglobina ( $11.46 \mathrm{gr} / \mathrm{dl}$ en ambas), reducción de $\beta 2 \mathrm{~m}(70.14 \%$ vs $65.19 \%, p=0.6$ ) y albúmina ( 3.60 vs 3.64 , gr/dl $p=0.4$ ) no fueron diferentes.

Se encontró una mayor presión en la línea venosa en la hemodiafiltración "en línea" (165.2 vs 149.9, $\mathrm{p}=0.017$ ), junto a una mayor hemoconcentración observada de forma subjetiva por Enfermería, si bien el volumen de líquido de reposición es significativamente mayor con hemodiafiltración "en línea". El tiempo de montaje y cebado es mayor en hemodiafiltración con reinfusión endógena HFR (2.28 vs 4.82, p=0.012), aunque no se observa más dificultad en el montaje de la técnica. La sesión la hemodiafiltración con reinfusión endógena requirió menos intervención de enfermería, ya que presenta menos variabilidad en los parámetros relacionados con la hemoconcentración, generando una menor cantidad de alarmas. No hubo diferencias significativas en la PCR como marcador inespecífico de inflamación, sin embargo, en el laboratorio experimental se observó una disminución de la actividad de los CD14++ y CDI6++ (monocitos pro-inflamatorios) en la hemodiafiltración con reinfusión endógena, y también presentó menos niveles de VEGF. 
Conclusiones: La hemodiafiltración con reinfusión endógena es una técnica que no plantea más dificultades técnicas que la hemodiafiltración "en línea" para enfermería, pues aunque el tiempo de montaje es mayor, requiere menor intervención posterior. No se encontraron diferencias en los parámetros técnicos y clínicos entre ambas técnicas. Sin embargo nuestros resultados preliminares muestran una disminución de la activación proinflamatoria de los monocitos en la hemodiafiltración con reinfusión endógena, con respecto a la hemodiafiltración "en línea".

A comparative analysis between online hemodiafiltration and hemodiafiltration with endogenous reinfusion: clinical, technical and inflammatory parameters.

\begin{abstract}
Introduction: Hemodialysis (HD) itself produces a chronic inflammatory process in the patient. Alternative techniques as hemodiafiltration "online" (OL-HDF) improve, among other things, this inflammatory profile. Nowadays, in this research line, new convective techniques are emerging. These techniques could be better than the HDF-OL. Hemodiafiltration with endogenous reinfusion or HFR-SUPRA (HFR) uses a two-chamber dialyzer plus a resin cartridge. In this technique the patient ultrafiltered liquid is subsequently reinfused to its regeneration in this resin cartridge.
\end{abstract}

The aim of our study was to evaluate the differences between the HFR and OL-HDF technique, in terms of clinical and inflammatory parameters and dialysis adequacy.

Materials and methods: Sixteen patients were included with a mean age of $61.6 \pm 14.5$ years. These patients were subjected to the two techniques for periods of eight weeks by the following scheme: HFR-1, HDFOL-1, HFR-2, HDF-0L-2. All patients underwent highflux HD in the 8 weeks prior. Technical (pressure in the arterial and venous lines, blood flow, treated liter, the infusion volume, and needs of heparin), clinical (systolic and diastolic blood pressure pre and post-dialysis, dry weight, interdialytic weight gain, incidents during the hemodialysis session) and laboratory parameters (elimination of B2m, hemoglobin, hematocrit, albumin) and inflammatory parameters were collected.

Results: There was no difference between the two techniques in the collected clinical parameters: systolic and diastolic pre and post-dialysis dry weight, interdialytic weight gain and incidents during the session. And even there were no significant differences in the needs of heparin or $\mathrm{Kt} / \mathrm{V}$. The hemoglobin values (both 11.46$),$ B2m reduction $(70.14 \%$ vs $65.19 \%, p$ $=0.6$ ) and albumin ( 3.60 vs $3.64, p=0.4$ ) were not significantly different.

It was found higher pressure in the venous line in the OL-HDF versus HFR ( 165.2 vs $149.9, \mathrm{p}=$ 0.017), together with greater hemoconcentration subjectively observed by Nursing. However, the volume of replacement fluid is significantly higher with the OL-HDF technique. The assembly time and priming were higher in the HFR technique (2.28 vs $4.82, p=$ 0.012 ), although greater difficulty in the assembly of the technique was not observed. During the session, the HFR technique requires less nursing intervention because it presents less variability in parameters related to hemoconcentration, generating fewer alarms. There were no significant differences in the PCR as nonspecific marker of inflammation. However, in the HFR technique a decrease in the activity of CD14 ++ and CD16 ++ (pro-inflammatory monocytes) and lower levels of VEGF were observed.

Conclusion: The HFR technique does not present more technical difficulties than the HDF-OL for nursing, for though the installation time is longer, requires less subsequent intervention.

No significant differences in the technical and clinical parameters between the two techniques were found. However, our preliminary results show a decrease of the proinflammatory activation of monocytes in the HFR with respect to the OL-HDF technique.

\section{Introducción}

El tratamiento con hemodiálisis (HD) produce "per sé" un proceso inflamatorio crónico en el paciente, siendo este hecho, causa bien conocida de aumento de la morbimortalidad tanto global como de causa cardiovascular ${ }^{1-3}$.

Este proceso inflamatorio mejora con la utilización de técnicas alternativas a la HD convencional como es la hemodiafiltración en línea (HDF-OL), que consigue mejorar los aclaramientos de toxinas urémicas al aumentar esta técnica el transporte convectivo ${ }^{4-6}$.

Para la realización de esta técnica, es necesario la utilización de una membrana de alto flujo, capaz de mantener durante la sesión una ultrafiltración elevada en ausencia de complicaciones técnicas en el circuito extracorpóreo, al mismo tiempo que se requiere el suministro de líquido de reposición para compensar el exceso 
de ultrafiltración. En la HDF-OL es el mismo líquido de diálisis generado por el monitor el utilizado para la reposición.

Recientemente están apareciendo nuevas técnicas convectivas como la hemodiafiltración con reinfusión endógena (HFR), que por su diseño podría mejorar los resultados de la HDF-OL ${ }^{7-9}$.

En esta técnica, el líquido de reposición utilizado es el mismo que previamente se ha ultrafiltrado, el cual se infunde en el circuito hemático una vez regenerado al hacerlo pasar por un cartucho que contiene una resina adsortiva en el que quedan adheridas las toxinas urémicas.

Para ello, la HFR utiliza un dializador de doble cámara. En la primera, fabricada con una membrana de muy alta permeabilidad, se lleva a cabo una ultrafiltración (convección), y en la segunda cámara, fabricada con una membrana de baja permeabilidad se produce una HD convencional (difusión).

El líquido ultrafiltrado en la primera cámara se hace pasar por el cartucho de resina adsortiva (adsorción), reiunfundiéndolo posteriormente en el circuito sanguíneo entre las dos cámaras del dializador.

Estas dos técnicas, HDF-OL y HFR, se fundamentan en la utilización de los principios de difusión y convección, siendo el elevado transporte convectivo, lo que las diferencia de la $\mathrm{HD}$ convencional. Sin embargo, la sangre del paciente es sometida a procesos y materiales diferentes en ambas técnicas, y en la HFR además de la difusión y convección, interviene un proceso de adsorción ${ }^{2,10}$.

El objetivo del presente estudio fue evaluar las diferencias entre la HDF-OL y la HFR en cuanto a parámetros clínicos, inflamatorios y de adecuación de la diálisis, así como respecto a su manejo por parte del personal de enfermería.

\section{Material y métodos}

\section{Pacientes y características}

Se incluyeron 16 pacientes, procedentes del mismo centro, que cumplían las siguientes características: Ser mayores de edad, que hubieran permanecido estables en hemodiálisis durante al menos 3 meses, portadores de una fístula arteriovenosa como acceso vascular que permitiera flujos elevados de sangre $(>350 \mathrm{ml} / \mathrm{min})$, con adecuada dosis de diálisis ( $\mathrm{Kt} / \mathrm{V}>1.3)$ y con valores de hemoglobina comprendidos entre 11.5 y 13 gr/dl.
Quedaron excluidos del estudio aquellos pacientes con antecedentes de neoplasias, marcadores virales positivos (hepatitis B y C y/o VIH), elevación de marcadores inflamatorios (proteína $\mathrm{C}$ reactiva $>7$ ) o hipoalbuminemia (albúmina $<3.5 \mathrm{gr} / \mathrm{dl}$ ).

Previamente a la obtención de muestras, se solicitó el consentimiento informado.

Durante todo el estudio, cada paciente se dializó el mismo número de horas por sesión que tenía establecido antes de la entrada en éste, y se mantuvieron estables las características de diálisis (conductividad $140 \mathrm{~m} / \mathrm{s}$, temperatura $36.5^{\circ}$, flujo del baño de diálisis $800 \mathrm{ml} / \mathrm{min}$, duración de la sesión, agujas de calibre 16G).

\section{Diseño del estudio}

Se diseñó un estudio prospectivo cruzado, en el que los pacientes siguieron el siguiente esquema: durante un mínimo de 8 semanas, los pacientes se sometieron a hemodiálisis con un dializador de alta permeabilidad, que se consideró como período de lavado. Tras este tiempo, y durante períodos consecutivos de 8 semanas, los pacientes se dializaron con diferentes técnicas: HFR - HDF-OL - HFR - HDF-OL, de manera que cada paciente fue su propio control.

\section{Material para la sesión}

- HFR: Monitores FórmulaTM 2000 o Fórmula Therapy y kit específico para esta técnica KIT SUPRA 17de (Bellco®).

- HDF-OL: Monitor Fresenius 5008. Dializador de helixona $1.8 \mathrm{~mm}$, líneas arterial y venosa, línea de infusión.

\section{Obtención de muestras}

Al final de cada período (incluido el período de lavado), se obtuvieron muestras de sangre en la segunda sesión de diálisis de la semana (miércoles o jueves según el turno del paciente). Asimismo, en los períodos de HFR se obtuvieron muestras del ultrafiltrado antes y después de pasar por la resina.

Estas muestras fueron adicionales a las que le correspondieran al paciente (analítica mensual o quincenal, $\mathrm{Kt} / \mathrm{V}$ etc.), y en ellas se midieron proteína $\mathrm{C}$ reactiva, albúmina, beta-2-microglobulina y hemoglobina. Además, en el laboratorio experimental se determinaron parámetros inflamatorios (monocitos CD14+CD16+) y de daño endotelial (Vascular Endotelial Growth Factor, VEGF). 
Parámetros clínicos y técnicos

Clínicos: Durante todas las sesiones de diálisis se midió la presión arterial sistólica (PAS) y presión arterial diastólica (PAD) prediálisis, después de la conexión, a intervalos horarios y post-diálisis. Asimismo, se registró el peso seco y la ganancia interdialítica. También se anotaron las incidencias, como los episodios de hipotensión arterial sintomática (que requieran infusión de fluidos o intervención del personal de enfermería), y de intolerancia al procedimiento (nauseas, vómitos, astenia, cefaleas, calambres y prurito).

Técnicos: Se recogieron la presión en las líneas arterial y venosa, la necesidad de heparina en el circuito, el flujo de sangre, los litros tratados y el volumen infundido.

\section{Cuestionario para Enfermería}

A todo el personal de enfermería participante en el estudio se les invitó a responder un cuestionario acerca de ambas técnicas: tiempo subjetivo de montaje (mayor/ menor/igual), minutos para el montaje, dificultad subjetiva (mayor/menor/igual), cantidad de suero cebado (mayor/menor/igual), número de alarmas detectadas (más/menos/las mismas), y si trabajar con la HFR les ha reportado nuevos conocimientos.

\section{Análisis estadístico}

Todos los datos se expresaron como medianas. La comparación entre las mismas se realizó con el test de Friedman para datos no paramétricos, aplicando la prueba de los rangos con signo de Wilcoxon para la comparación entre los diferentes períodos entre sí.

El análisis se llevó a cabo utilizando el programa estadístico SPSS 15.0. Se consideró que existen diferencias estadísticamente significativas cuando $p<0,05$.

\section{Resultados}

No se encontraron diferencias estadísticamente significativas entre ambas técnicas en cuanto a los parámetros clínicos recogidos. Los valores de hemoglobina (11.46 gr/dl en ambas), reducción de B2m $(70.14 \%$ vs $65.19 \%$ ) y albúmina (3.60 vs $3.64 \mathrm{gr} / \mathrm{dl}$ ) no fueron significativamente diferentes. Tampoco hubo diferencias en cuanto a la eficacia de la diálisis, PASpre, PADpre y PADpost, dosis de heparina, Peso seco y ganancia entre ambas técnicas, tal como apreciarse en la Tabla I. Si se objetivó diferencias significativas entre ambas técnicas en la PASpost, aunque sin relevancia clínica.
Tabla 1. Valores de la presión arterial Pre y Post $(\mathrm{mmHg})$ periodo con cada una de las técnicas. Kt/V, dosis de heparina (UI), peso seco (Kgr) y ganancia de peso (mgr) entre ambas técnicas.

Se encontró una mayor presión en la línea venosa en la HDF-0L frente a HFR (165.2 vs. $149.9, \mathrm{mmHg}$, $\mathrm{p}=0.017$ ), junto a una mayor hemoconcentración observada de forma subjetiva por enfermería, si bien el volumen infundido también fue mayor, tal como puede apreciarse en la figura 1.

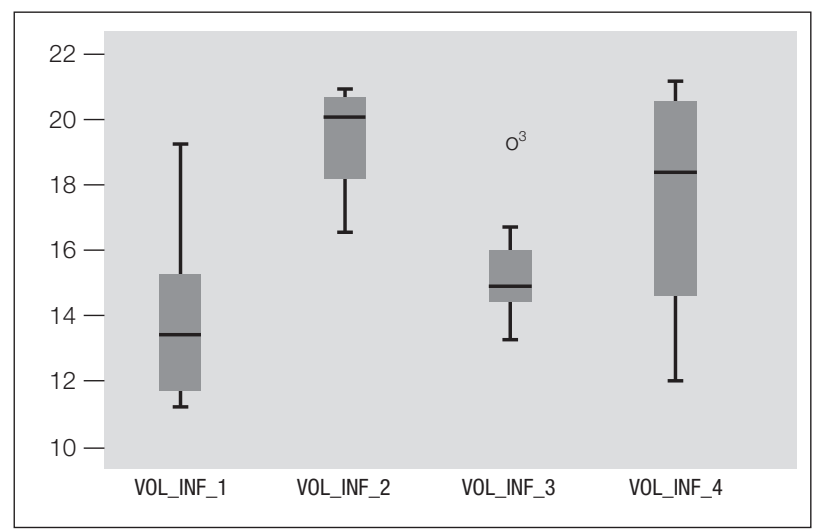

Figura 1. Comparación del Volumen de Infusión o Intercambio entre los 4 periodos de estudio con ambas técnicas en litros (VOL_INF_I $=$ HFR, VOL_INF_2 = HDF-OL, VOL_INF_3 = HFR, VOL_INF_2 = HDF-OL).

En cuanto a los parámetros de inflamación y disfunción endotelial, determinados por la concentración de monocitos CD14+CD16++ y el VEGF, respectivamente, se observó una mejoría de ambos parámetros en los períodos de HFR con respecto a HDF-OL, como puede observarse en la figura 2.

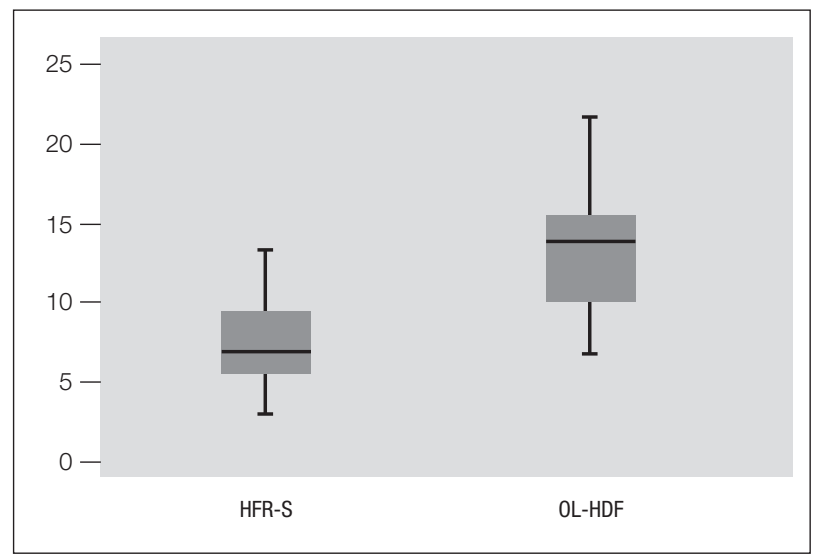

Figura 2. Comparación de la activación de monocitos pro-inflamatorios CD14+CD16++ entre ambas técnicas (HFR vs HDF-OL). 
El tiempo de montaje y cebado fue mayor en HFR (2.28 vs. 4.82 minutos, $p=0.012$ ) sin haber diferencias en cuanto a la dificultad de montaje. En la figura 3 está representada la opinión de las/os enfermeras/os respecto a si el montaje y cebado de la técnica de la HFR con respecto a la HDF-OL.

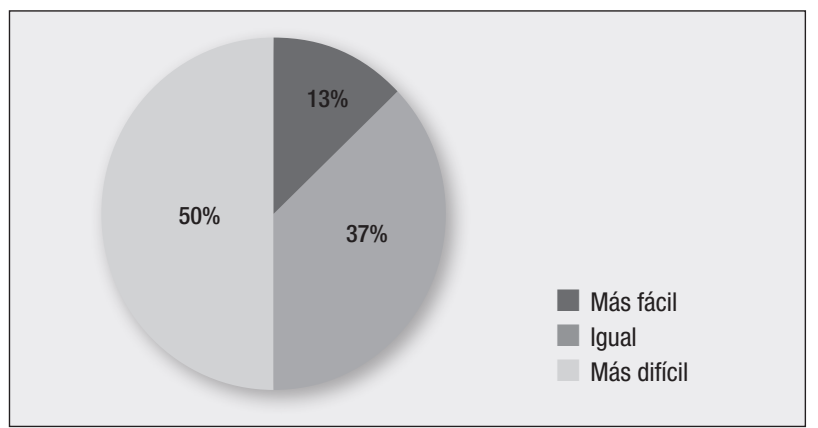

Figura 3. Opinión de enfermería sobre la dificultad de montaje y cebado de la HFR respecto a la técnica HDF-OL.

Sin embargo, durante la sesión, la HFR requiere menos intervenciones por parte de enfermería, ya que presenta menos variabilidad en los parámetros y por lo tanto genera menor frecuencia de alarmas, como puede apreciarse en la figura 4.

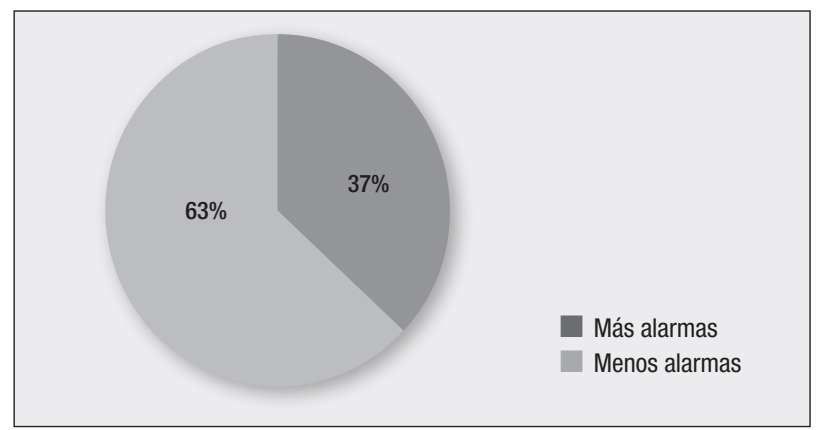

Figura 4. Opinión de enfermería sobre el número de alarmas en HFR, respecto a HDF-OL.

\section{Discusión}

El objetivo del presente estudio fue evaluar las diferencias entre la HDF-OL y la HFR en cuanto a parámetros clínicos, inflamatorios y de adecuación de la diálisis, así como respecto a su manejo por parte del personal de enfermería. Nuestros resultados muestran una buena tolerancia hemodinámica de los pacientes durante las sesiones tanto de HFR como de HDF-OL, sin diferencias significativas en los parámetros clínicos registrados. Esto está en consonancia con estudios previosll-13, que refieren mejor tolerancia hemodinámica en pacientes tratados con técnicas depurativas convectivas.
Por otro lado, encontramos diferencias en las presiones de la línea venosa del circuito, siendo significativamente mayor en la HDF-OL que en la HFR. Esto sin duda es debido a la mayor hemoconcentración que se produce en el circuito de HDF-OL, lo cual se constata por el aumento significativo del volumen total de reinfusión que se alcanza con esta técnica.

Frente a un estudio previo al nuestro ${ }^{14}$, en el que se comparaban los cambios bioquímicos que se experimentan en el paciente con estas dos técnicas, y en el que concluyen que con la HFR se mejoran significativamente los parámetros nutricionales, y que la eliminación de $\beta_{2}$ microglobulina en la HFR también es significativamente mayor en la HFD-OL respecto a la HFR, en nuestro estudio no encontramos diferencias en estos parámetros. Sin duda esta discrepancia se debe a la limitación del estudio referido al haberse obtenido sus resultados solamente con los datos de una sesión, circunstancia esta que destacan en sus conclusiones, planteando la necesidad de confirmar sus resultados con estudios más prolongados en el tiempo.

Respecto a la mejoría en los parámetros inflamatorios y de daño endotelial que observamos en la HFR respecto de la HDF-OL, y a pesar de la discrepancia con el estudio de Panichi $^{2}$, que concluye que no hay diferencias significativas en la reducción de los parámetros inflamatorios, junto a los datos que sugieren una mejoría en la tolerancia hemodinámica durante las sesiones, pensamos que están relacionados tal y como se afirma en un estudio de Cobo J.L. et $\mathrm{al}^{15}$, que concluye que la HFR es una técnica que presenta un mayor grado de biocompatibilidad, seguramente debido al origen endógeno del líquido de reposición que se utiliza en la misma. Esto debe ser confirmado, tal y como sugiere Panichi ${ }^{2}$ en sus conclusiones, y debe ser objeto de estudios más prolongados en el tiempo.

En cuanto a la dificultad técnica que plantea para el personal de enfermería cada técnica, podemos afirmar que aunque en la HFR se emplea un tiempo de montaje y cebado mayor que en la HDF-OL, esto se compensa al registrarse menos eventos y alarmas durante la sesión de HFR, sin duda debido a la mejor tolerancia hemodinámica intrasesión, tal y como se ha descrito, y a la presencia en esta técnica de presiones en el circuito más estables y moderadas que en la HFD-0L, condicionadas por el menor volumen de ultrafiltración que se registra en la HFR.

Por tanto, podemos concluir que la HFR es una técnica que no plantea más dificultades técnicas que la HDF-0L, y que aunque no se han encontrado diferencias significativas en los parámetros técnicos y clínicos, con la HFR 
se obtiene una mejoría en los parámetros inflamatorios y de daño endotelial frente a la HDF-OL, con los beneficios clínicos que esto conlleva para nuestros pacientes.

\section{Agradecimientos}

En primer lugar a la Dra. Elvira Esquivias de Motta, Nefróloga, por su disposición, colaboración y ánimo desde el comienzo del estudio de investigación hasta su finalización. A Rodolfo Crespo y Mateo Alcántara por su colaboración en la línea de investigación.

A todo el personal del Centro de Diálisis por su paciencia y buen hacer para finalizar el proyecto.

\section{Bibliografía}

1. Zimmermann J, Herrlinger S, Pruy A et al. Inflammation entrances cardiovascular risk and mortality in hemodialysis Patiens. Kidney Int. 1999; 55: 648658.

2. Panichi $V$, et al. Effects on inflammatory and nutritional markers of haemodiafiltration with online regeneration of ultrafiltrate (HFR) vs online haemodiafiltration: a cross-over randomized multicentre trial. Nefrol. Dial. Transpl. 2006; 21: 756-762.

3. Stenvinkel $P$, Heinburger 0 , Paultre $F$, et al. Strong associations between malnutrition, inflammation and aterosclerosis in chronic renal failure. Kidney Int. 1999; 55: 1899-911.

4. Merino A, Portoles J, Selgas R, et al: Effect of different dialysis modalities on microinflammatory status and endothelial damage. Clin J Am Soc Nephrol 2010; 5: 227-234.

5. Ok E, Asci G, Toz H, et al: Mortality and cardiovascular events in online haemodiafiltration (OLHDF) compared with high-flux dialysis: results from the Turkish OL-HDF Study. Nephrol Dial Transplant 2013; 28: 192-202.

6. Ariza F, Merina A, Carracedo J, Álvarez-Lara MA, Crespo R, Ramírez R, Martín-Malo A Aljama P. PostDilution High Convective Transport Improves Microinflammation and Endothelial Dysfunction Independently of the Technique. Bood Purification 2013, 35(4): 270-278.
7. De Francisco ALM, Ghezzi PM, Brendolan A, et al. Hemodiafiltration with online regeneration of the ultrafiltrate. Kidney Int. 2000; 58: 566-71.

8. Caló LA, Naso A, Carraro G, et al. Effect of haemodiafiltration with online regeneration of ultrafiltrate on oxidative stress in dialysis patients. Nefrol. Dial. Transpl. 2007; 22: 1413-9.

9. Álvarez Serrano $M^{a} D$, et al: Nuevas terapias en hemodiálisis: HFR aequilibrium. Rev Soc. Esp. Enferm. Nefrol. 2010; 13 (4):247-251.

10. Ghezzi PM, Gervasio R, Tessore V, Sartoris AM, Botella J. Hemodiafiltracion without replacement fluid: an experimental study ASAIO Journal 1992; 38: 61-65.

11. Ramírez R, Martín-Malo A, Aljama P. Evolution of the concept of biocompatibility and the cardioprotective effect of on-line hemodiafiltration. Contrib Nephrol. 2011;175:110-116.

12. ACanaud B, Bragg-Gresham JL, Marshall MR. Mortality risk for patients receiving hemodiafiltration versus hemodialysis: European results from the D0PPS. Kidney Int 2006; 69: 2087-2093.

13. Grooteman $M$, van den Dorpel $M$, Bots $M$, et al, for the CONTRAST Investigators: Effect of online hemodiafiltration on all-cause mortality and cardiovascular outcomes. J Am Soc Nephrol 2012; 23: 1087-1096.

14. Menezo Viadero R, et al: Hemodiafiltración en línea Vs hemodiafiltración con reinfusión endógena: estudio comparativo de cambios bioquímicos. Enfermería Nefrológica. 2012; 15(supl.1):54.

15. Cobo Sánchez JL, et al: Estudio comparativo de biocompatibilidad entre la hemodiafiltración en línea y la hemodiafitración con reinfusión endógena. Enfermería Nefrológica.2012;15(4):271-276. 